\title{
Celiprolol reduces oxidative stress and attenuates left ventricular remodeling induced by hypoxic stress in mice
}

\author{
Satoshi Nishioka ${ }^{1}$, Toshitaka Yoshioka ${ }^{1}$, Atsuo Nomura ${ }^{2}$, Ryuji Kato ${ }^{2}$, Masatoshi Miyamura ${ }^{3}$, \\ Yoshikatsu Okada ${ }^{4}$, Nobukazu Ishizaka ${ }^{3}$, Yasuo Matsumura ${ }^{1}$ and Tetsuya Hayashi ${ }^{2}$
}

We have previously reported that intermittent hypoxic stress, which is relevant to sleep apnea syndrome (SAS), increases oxidative stress and induces left ventricular (LV) remodeling. Celiprolol, a $\boldsymbol{\beta}_{1}$-selective adrenoreceptor blocker, is known to have not only an antihypertensive effect but also an antioxidant effect through releasing nitric oxide. The aim of this study was to examine the hypothesis that celiprolol might ameliorate the LV remodeling induced by intermittent hypoxia through its antioxidant effect. Male C57BL/6J mice ( 8 weeks old) were exposed to intermittent hypoxia ( $30 \mathrm{~s}$ of $5 \%$ oxygen followed by $30 \mathrm{~s}$ of $21 \%$ oxygen) for $8 \mathrm{~h} \mathrm{day}^{-1}$ during the daytime for 10 consecutive days or were maintained under normoxic conditions. Animals were treated with either celiprolol ( $100 \mathrm{mg} \mathrm{kg}^{-1}$ day $^{-1}$ by gavage) or vehicle. Hypoxic stress caused fluctuations in blood pressure (BP), an increase in the mean cardiomyocyte diameter, perivascular fibrosis and a decrease in endothelial nitric oxide synthase (eNOS) expression. These changes were associated with increased levels of 4-hydroxy-2-nonenal protein, superoxide, tumor necrosis factor- $\alpha$ mRNA and brain natriuretic peptide mRNA in the LV myocardium. Celiprolol significantly suppressed BP fluctuation, restored eNOS expression and reduced oxidative stress and superoxide production, thus ameliorating hypoxia-induced LV remodeling in mice. These findings suggest that treatment with celiprolol might prevent cardiovascular events in borderline hypertensive patients with SAS.

Hypertension Research (2013) 36, 934-939; doi:10.1038/hr.2013.60; published online 20 June 2013

Keywords: celiprolol; intermittent hypoxia; oxidative stress

\section{INTRODUCTION}

Sleep apnea syndrome (SAS) is a risk factor for cardiovascular disease, being associated with hypertension, coronary artery disease and stroke. ${ }^{1-4}$ Major pathophysiological features of SAS include the production of reactive oxygen species (ROS) and inflammatory cytokines induced by intermittent hypoxia., ${ }^{5,6}$ It has been reported that ROS can cause endothelial dysfunction, cardiomyocyte hypertrophy and interstitial fibrosis. ${ }^{7}$ We have previously demonstrated that gp91 phox $_{\text {-containing }} \mathrm{NADPH}$ (nicotinamide adenine dinucleotide phosphate reduced) oxidase has a crucial role in the development of left ventricular (LV) remodeling induced by intermittent hypoxia in mice. ${ }^{8}$ In addition, hypoxic stress enhances atherosclerosis and LV remodeling in apolipoprotein E-knockout mice, while treatment with an angiotensin II receptor blocker reduces oxidative stress and ameliorates interstitial fibrosis in the LV myocardium. ${ }^{9}$ In clinical studies, diastolic dysfunction and a decrease in the myocardial contractile reserve, suggesting myocardial degeneration and/or interstitial fibrosis, have been observed in patients with moderate-to-severe SAS. ${ }^{10}$ In addition, both blood pressure (BP) and sympathetic nerve activity are increased during sleep in SAS patients. ${ }^{11}$

Some $\beta_{1}$-selective adrenoreceptor blockers are known to have not only an antihypertensive effect but also an antioxidant effect. Celiprolol is a $\beta_{1}$-selective adrenoreceptor blocker with intrinsic sympathomimetic activity that also promotes the release of nitric oxide (NO) and decreases ROS production. ${ }^{12-14}$ However, the effect of celiprolol on LV remodeling induced by intermittent hypoxia remains unclear. Thus, the aim of this study was to examine the hypothesis that celiprolol might ameliorate LV remodeling induced by intermittent hypoxia through its antioxidant effect.

\section{METHODS}

Experimental protocol

Eight-week-old male C57BL/6J mice $(n=60)$ were purchased from Clea Japan, Inc. (Osaka, Japan) for this study. The mice were exposed to a 12-h light-dark cycle, given free access to tap water and fed standard chow ad libitum.

${ }^{1}$ Laboratory of Pathological and Molecular Pharmacology, Osaka University of Pharmaceutical Sciences, Osaka, Japan; ${ }^{2}$ Laboratory of Cardiovascular Pharmacotherapy and Toxicology, Osaka University of Pharmaceutical Sciences, Osaka, Japan; ${ }^{3}$ Department of Internal Medicine III, Osaka Medical College, Osaka, Japan and ${ }^{4}$ Department of Pathology, Osaka Medical College, Osaka, Japan

Correspondence: Dr T Hayashi, Laboratory of Cardiovascular Pharmacotherapy and Toxicology, Osaka University of Pharmaceutical Sciences, Nasahara 4-20-1, Takatsuki, Osaka 569-1094, Japan.

E-mail: hayashi@gly.oups.ac.jp

Received 6 December 2012; revised 25 March 2013; accepted 11 April 2013; published online 20 June 2013 
The hypoxic stress group was housed in a chamber and exposed to intermittent hypoxia ( $30 \mathrm{~s}$ of $4.5-5.5 \%$ oxygen followed by $30 \mathrm{~s}$ of $21 \%$ oxygen) for $8 \mathrm{~h} \mathrm{day}^{-1}$ during the daytime for 10 consecutive days (IH mice). Control animals were housed in identical cages in the same room and were exposed to the same ambient noise and lighting conditions as the IH mice. The animals were administered celiprolol $\left(100 \mathrm{mg} \mathrm{kg}^{-1} \mathrm{day}^{-1}\right)$ or its vehicle by oral gavage. After the 10-day experimental period, cardiac catheterization was performed under anesthesia with intraperitoneal pentobarbital sodium $\left(50 \mathrm{mg} \mathrm{kg}^{-1}\right)$ to measure the biventricular systolic pressure. ${ }^{9}$ Then the heart was excised, and the upper half was used for light microscopy, while the LV free wall was excised for immunohistochemistry and the reverse transcription PCR. The experimental protocol and handling of animals during experiments were approved by the Experimental Animal Research Committee of Osaka University of Pharmaceutical Sciences.

\section{Monitoring of $\mathrm{BP}$ and heart rate (HR)}

The arterial pressure was monitored during hypoxic stress by using a Data-quest IV radiotelemetry system (Data Sciences International, St Paul, MN, USA). Under anesthesia, a thoracic aortic catheter was implanted in each mouse and was attached to a PA-C10 radiotelemetry transmitter. The transmitter was secured in a subcutaneous pocket for the duration of the experiment. After 1 week for recovery from surgery, the BP and HR were recorded throughout the experiment.

\section{Histological examination}

Isolated ventricular tissues were fixed in $10 \%$ formaldehyde, embedded in paraffin and cut into $4-\mu \mathrm{m}$ sections. To evaluate the mean cardiomyocyte diameter, the shortest diameter of each nucleated cardiomyocyte was measured on transverse sections stained with hematoxylin-eosin under a light microscope at a $\times 400$ magnification. ${ }^{10}$ After staining with Sirius Red, color images of each section were taken with a digital camera (Fujix Digital Camera HC-300Z; Fujifilm, Tokyo, Japan) mounted on a Nikon Microphot-FXA (Nikon, Tokyo, Japan), and the percentage of the area of interstitial fibrosis was calculated by the previously described method. ${ }^{9}$

\section{Immunohistochemistry for 4-hydroxy-2-nonenal (4-HNE), macrophages, endothelial nitric oxide synthase (eNOS) and arginase II}

Immunohistochemical staining was performed as described previously ${ }^{9}$ Briefly, sections cut from paraffin blocks were incubated with antibodies targeting 4-HNE (No. MHN-20; Japan Institute for the Control of Aging, Shizuoka, Japan), macrophages (anti-CD68, No.ab125212; Abcam, Tokyo, Japan), arginase II (H-64, Santa Cruz Biotechnology, Dallas, TX, USA) or eNOS (BD Biosciences, San Jose, CA, USA). Then the percentage of the area of 4-HNE, eNOS or arginase II staining was measured by the method described previously. ${ }^{10}$

\section{Detection of superoxide in the LV myocardium}

To evaluate in situ superoxide production, fresh frozen sections of LV myocardium were stained with dihydroethidium (DHE; Molecular Probes, Eugene, OR, USA). ${ }^{15}$ Frozen sections $(20-\mu \mathrm{m}$ thick) were covered with $10 \mu \mathrm{m}$ DHE solution and incubated at $37^{\circ} \mathrm{C}$ for $30 \mathrm{~min}$ in a humidified chamber with protection from light. Then the sections were observed under a fluorescence microscope (Keyence BZ-8000; Osaka, Japan), and the fluorescence intensity of DHE was quantified with NIH Image 1.61 software (National Institute of Mental Health, Bethesda, MD, USA) and expressed as a percentage of the total cross-sectional area. ${ }^{16}$

\section{Quantitative real-time reverse transcription PCR}

Total RNA was extracted from myocardial tissue using an RNeasy mini kit (Qiagen, Valencia, CA, USA). Reverse transcription was performed with random hexamers and superscript reverse transcriptase (Invitrogen, Carlsbad, CA, USA). Real-time quantitative PCR was done with an ABI Step One sequence detector (PE Applied Biosystems, Foster City, CA, USA), using a Taqman probe and primers for the target mRNA (Ma00443258 for tumor necrosis factor- $\alpha$ (TNF- $\alpha)$ ) purchased from Applied Biosystems. The reaction conditions were $50{ }^{\circ} \mathrm{C}$ for $2 \mathrm{~min}$ and $95^{\circ} \mathrm{C}$ for $10 \mathrm{~min}$, followed by 40 cycles of $95^{\circ} \mathrm{C}$ for $15 \mathrm{~s}$ and $60^{\circ} \mathrm{C}$ for $1 \mathrm{~min}$. The amount of target mRNA was normalized for that of $18 \mathrm{~s}$ mRNA. ${ }^{17}$

\section{Statistical analysis}

Results are expressed as the mean \pm s.e.m. Variables were compared between groups by one-way analysis of variance followed by the Tukey-Kramer multiple comparison test, and $P<0.05$ was considered to indicate significance.

\section{RESULTS}

Body weight, heart weight and hemodynamics

There were no significant differences in body weight, heart weight, HR and biventricular systolic and LV end-diastolic pressures among the groups (Table 1).

\section{Changes in BP during intermittent hypoxia}

During the hypoxic interval, there was an instantaneous surge in BP associated with an increased HR, and BP returned to baseline after a few seconds. Reoxygenation with room air caused a similar BP surge and tachycardia for several seconds, after which the BP and HR returned to the basal levels. These BP fluctuations, associated with an increased s.d. of the mean BP, were observed during intermittent hypoxia throughout the experiments (Figure 1). The mean BP showed elevation from 3 days after the initiation of hypoxic stress and was significantly elevated on the tenth experimental day. Treatment with celiprolol suppressed these BP and HR fluctuations, although the mean BP was elevated during hypoxic stress (Figure 2).

\section{Histological findings}

Exposure to intermittent hypoxia led to an increase in the mean cardiomyocyte diameter, myofiber disarray and interstitial fibrosis in the LV myocardium. In particular, the percentage of the area of perivascular fibrosis was significantly increased (Figure 3). A small number of CD68 $(+)$ macrophages were observed in the vicinity of arteries.

Treatment with celiprolol prevented these histological changes caused by hypoxic stress.

\section{Superoxide and 4-HNE}

IH mice showed an increase in superoxide production (detected by DHE labeling) and an increase in 4-HNE-modified protein adducts in the LV myocardium. Treatment with celiprolol significantly

Table 1 Effect of hypoxia on body weight, heart weight and hemodynamics

\begin{tabular}{lccccc}
\hline & \multicolumn{2}{c}{ Normoxia } & & \multicolumn{2}{c}{ Hypoxia } \\
\cline { 2 - 3 } \cline { 5 - 6 } Parameters & Vehicle & Celiprolol & & Vehicle & Celiprolol \\
\cline { 2 - 3 } BW, g & $22.0 \pm 0.8(8)$ & $23.6 \pm 0.5(8)$ & $22.1 \pm 0.5(13)$ & $22.1 \pm 0.4(13)$ \\
HW, mg & $111 \pm 3(8)$ & $112 \pm 5(8)$ & & $106 \pm 3(13)$ & $105 \pm 3(13)$ \\
HW/BW, mgg & $5.1 \pm 0.2(8)$ & $4.8 \pm 0.2(8)$ & $4.8 \pm 0.1(13)$ & $4.7 \pm 0.2(13)$ \\
HR, b.p.m. & $461 \pm 28(8)$ & $492 \pm 34(5)$ & $517 \pm 27(7)$ & $425 \pm 29(10)$ \\
RVsys, mm Hg & $26 \pm 4(7)$ & $33 \pm 4(5)$ & & $33 \pm 6(8)$ & $27 \pm 2(10)$ \\
LVsys, mm Hg & $101 \pm 2(7)$ & $102 \pm 3(5)$ & & $108 \pm 3(8)$ & $103 \pm 3(8)$ \\
LVed, mm Hg & $7 \pm 4(7)$ & $5 \pm 1(5)$ & & $9 \pm 4(8)$ & $5 \pm 1(7)$
\end{tabular}

Abbreviations: BW, body weight; HW, heart weight; HR, heart rate; LVed, left ventricula end-diastolic pressure; LVsys, LV systolic pressure; RVsys, right ventricular systolic pressure. Values are means \pm s.e.m. Number of mice in parenthesis. 

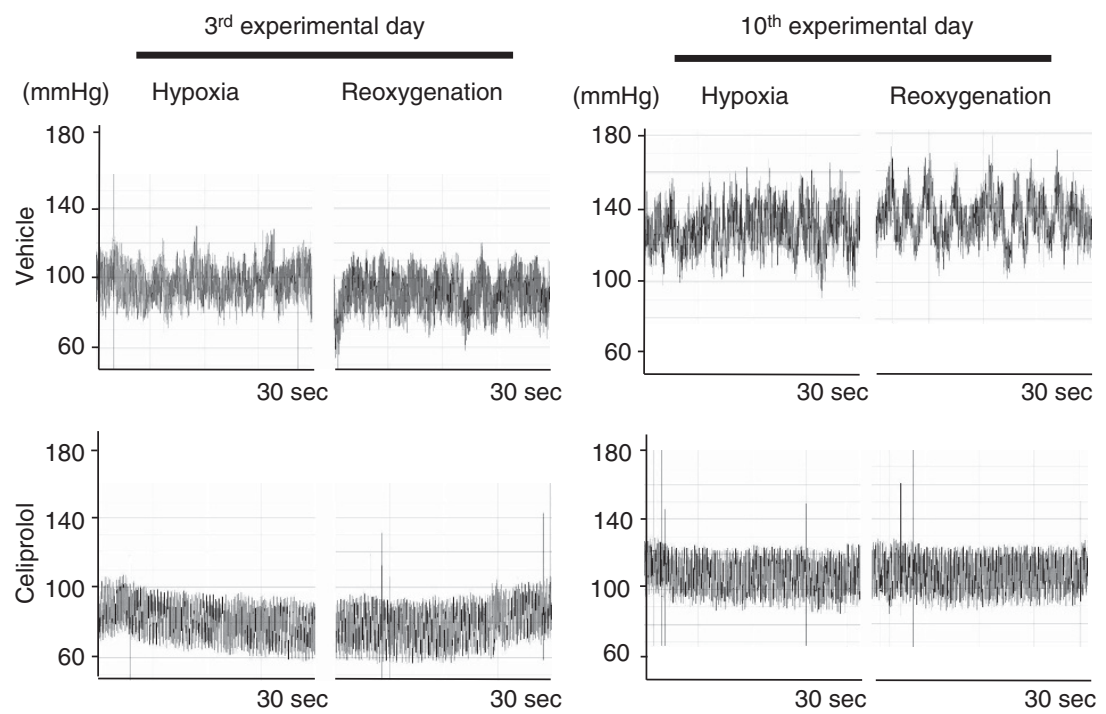

Figure 1 Monitoring of BP by telemetry. Arterial pressure was monitored in mice during hypoxic stress using a Data quest IV radiotelemetry system (Data Sciences International). BP and HR fluctuations were observed during intermittent hypoxia, while celiprolol suppressed these fluctuations. A full color version of this figure is available at the Hypertension Research journal online.

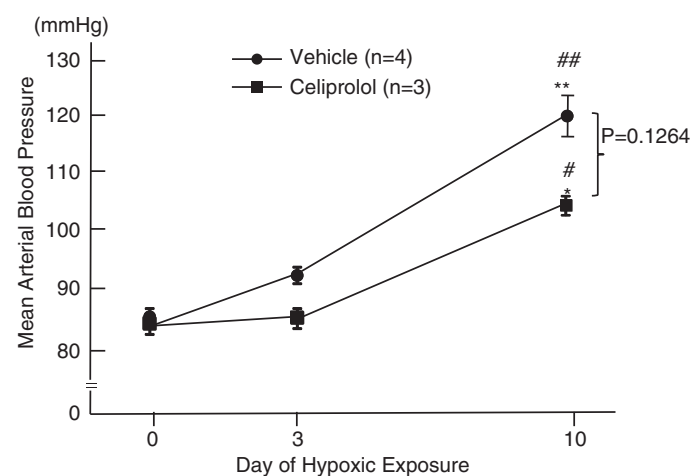

Figure 2 Mean arterial BP during intermittent hypoxia monitored by telemetry. The mean BP showed elevation from 3 days after the initiation of hypoxic stress, and was significantly elevated on the tenth experimental day. ${ }^{*} P<0.05,{ }^{* *} P<0.01$ vs. Day 0; ${ }^{\#} P<0.05,{ }^{\#} P<0.01$ vs. Day 3.

suppressed superoxide production and the accumulation of 4-HNE adducts in the LV myocardium (Figures 4 and 5a).

\section{TNF- $\alpha$ and brain natriuretic peptide (BNP)}

IH mice showed an increase in the expression of TNF- $\alpha$ and BNP mRNA, whereas these changes were significantly suppressed by treatment with celiprolol (Figures $5 \mathrm{~b}$ and $\mathrm{c}$ ).

\section{eNOS and arginase II}

Both eNOS and arginase II have the same substrate. Arginase metabolizes L-arginine to urea and ornithine, and it competes directly with eNOS for L-arginine. The expression of eNOS was decreased in the LV myocardium of IH mice, whereas the expression of arginase II was increased. These changes were less marked after treatment with celiprolol (Figure 6).

\section{DISCUSSION}

The major features of SAS include sympathetic activation, production of ROS and inflammation., ${ }^{5,6}$ In the present study, celiprolol suppressed the fluctuation of $\mathrm{BP}$ and $\mathrm{HR}$ during intermittent hypoxia, reduced superoxide production and decreased the expression of TNF- $\alpha$ and BNP mRNA in the LV myocardium, consequently attenuating the histological changes caused by chronic intermittent hypobaric hypoxia.

Although treatment with celiprolol at $100 \mathrm{mg}$ day $^{-1}$ did not affect the hemodynamics at the time of killing, this agent significantly reduced oxidative stress and superoxide production. We previously reported that oxidative stress mediated by NADPH oxidase has an important role in LV remodeling caused by intermittent hypoxia. ${ }^{8,17,18}$ Therefore, celiprolol might attenuate the perivascular fibrosis and myocardial degeneration observed in mice exposed to hypoxia by reducing the impact of oxidative stress on the LV myocardium.

In the present study, celiprolol suppressed the intermittent hypoxia-induced changes in arginase II and eNOS expression. It is well known that $\mathrm{NO}$ is produced from L-arginine by NOS, while arginase competes with NOS for L-arginine to produce urea and L-ornithine. Increased arginase expression has been observed in a number of conditions, including vascular dysfunction, ${ }^{19}$ pulmonary hypertension, ${ }^{20}$ coronary artery dysfunction ${ }^{21}$ and hypertension due to chronic hypobaric hypoxia. ${ }^{22}$ The upregulation of arginase activity might reduce the availability of L-arginine for NOS, leading to a decrease in NO production. ${ }^{23}$ Thus, the downregulation of arginase II observed in our study might be important for the vasodilatory action of celiprolol. Kobayashi et al. ${ }^{24}$ reported that celiprolol might act on eNOS through the PI3K (phosphatidylinositol 3'-kinase)-Akt signaling pathway, because phosphorylation of eNOS after treatment with celiprolol was attenuated by wortmannin in deoxycorticosterone acetate-salt hypertensive rats.

Sympathetic activity during sleep is known to be increased in SAS. Patients with congestive heart failure, especially those with central SAS, have a poor prognosis and show elevated plasma norepinephrine levels compared with those without SAS. ${ }^{25}$ In patients with obstructive SAS, muscle sympathetic nerve activity and plasma catecholamine levels are increased, associated with systemic and/or pulmonary hypertension. ${ }^{26,27}$ It seems that celiprolol decreases sympathetic tone and reduces BP fluctuation, which might be 


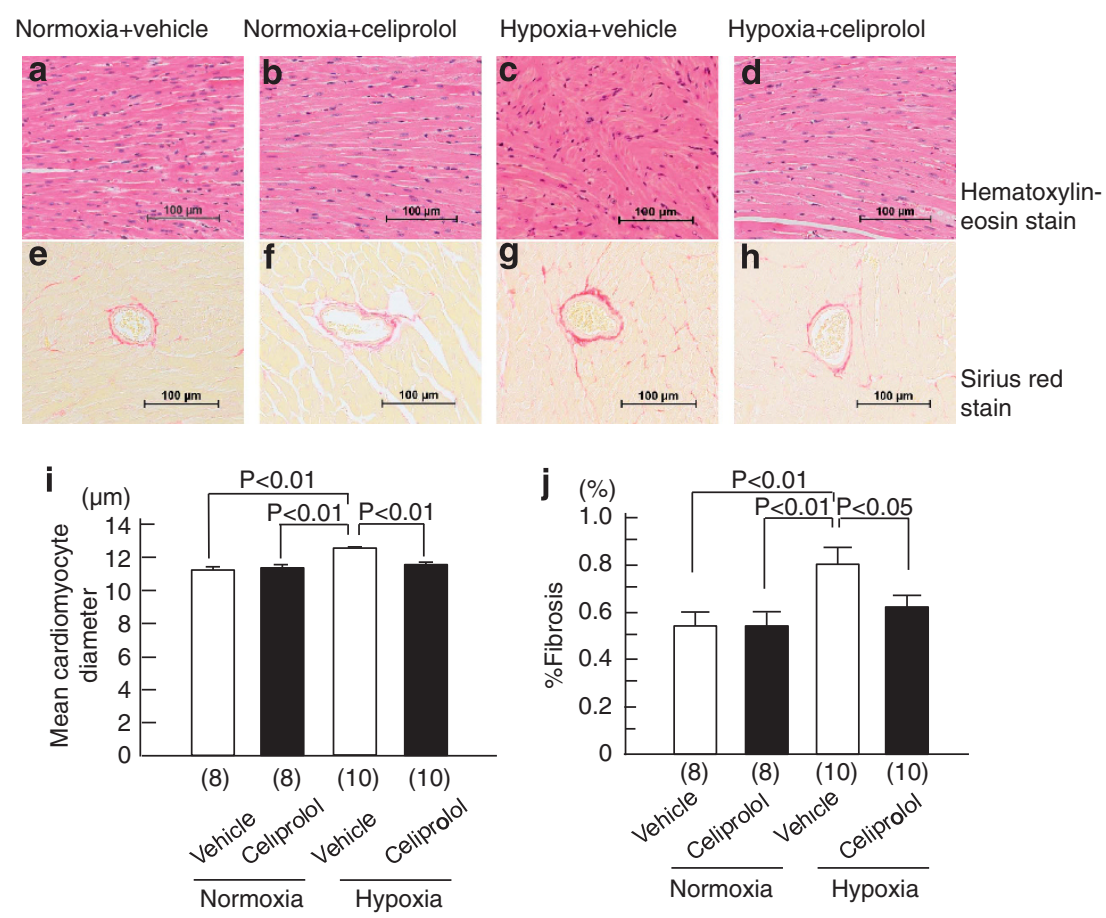

Figure 3 Representative light micrographs of LV myocardium (a-h). Hematoxylin-eosin stains (original magnification $\times 400$, (a-d)). Sirius red stain (original magnification $\times 200$, $(\mathbf{e}-\mathbf{h})$ ). Effect of intermittent hypoxic stress on the mean diameter of cardiomyocytes (i) and perivascular fibrosis (j) in the LV myocardium. The mean cardiomyocyte diameter and the percentage of the area of perivascular fibrosis were significantly increased in mice exposed to intermittent hypoxia, while this change was significantly suppressed by treatment with celiprolol. Values are the mean \pm s.e.m.
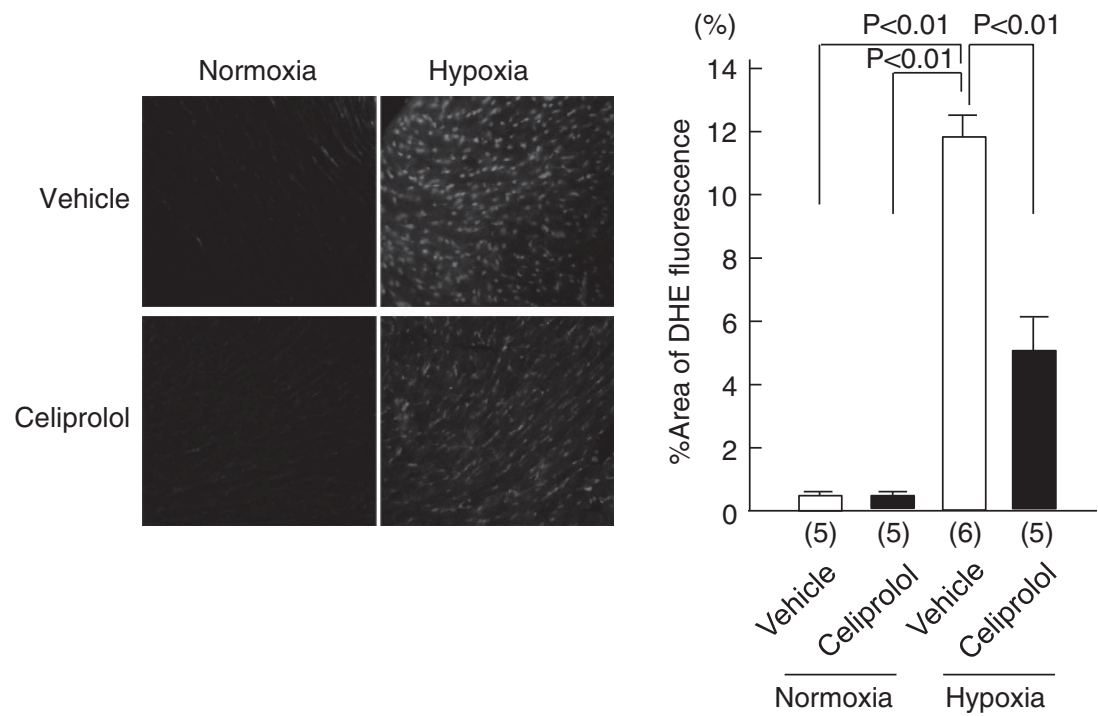

Figure 4 Effect of hypoxic stress on expression of DHE in the LV myocardium. DHE staining was significantly increased in mice exposed to intermittent hypoxia, suggesting an increase in superoxide production. Treatment with celiprolol significantly decreased superoxide production in the LV myocardium. Values are the mean \pm s.e.m. A full color version of this figure is available at the Hypertension Research journal online.

important factors mediating its cardioprotective effect observed in the present study. Further studies, with longer observation periods, are required to evaluate the effect of celiprolol to prevent hypertension in the future.

Intermittent hypoxia is known to increase the levels of various inflammatory factors. Li et al. ${ }^{28}$ reported that the serum levels of TNF- $\alpha$, interleukin- 8 and interleukin- 6 , as well as the phosphorylation of nuclear factor- $\mathrm{B}$ in endothelial cells, were increased over time, which might be related to the occurrence of cardiovascular events and hypertension due to intermittent hypoxia. Increased levels of TNF- $\alpha$ and oxidative stress promote the activation of nuclear factor- $\kappa \mathrm{B}$ and the development of cardiac hypertrophy. It has also been reported that intermittent hypoxic stress significantly increases transforming growth factor- $\beta$ (TGF- $\beta$ ), which has a major 

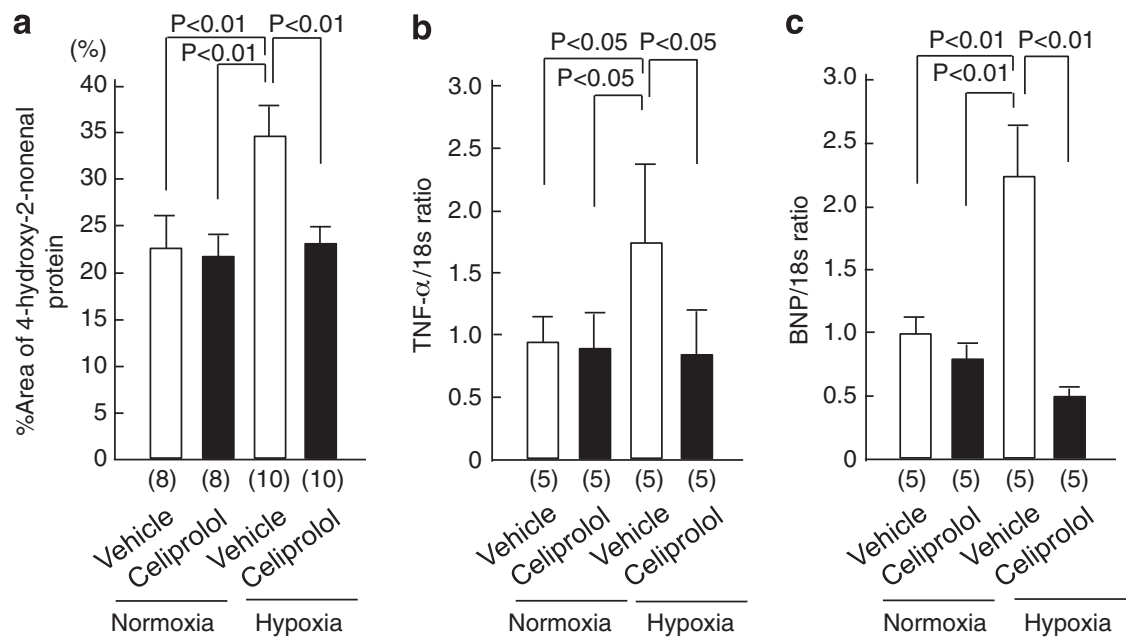

Figure 5 Quantitative data of immunohistochemistry and reverse transcription PCR. Intermittent hypoxia significantly increased 4-HNE-modified protein adducts (a), and the expression of TNF- $\alpha$ and BNP mRNA (b, c) in the LV myocardium. Celiprolol reduced oxidative stress and suppressed the intermittent hypoxia-induced increase in myocardial levels of TNF- $\alpha$ and BNP mRNA. Values are the mean \pm s.e.m.
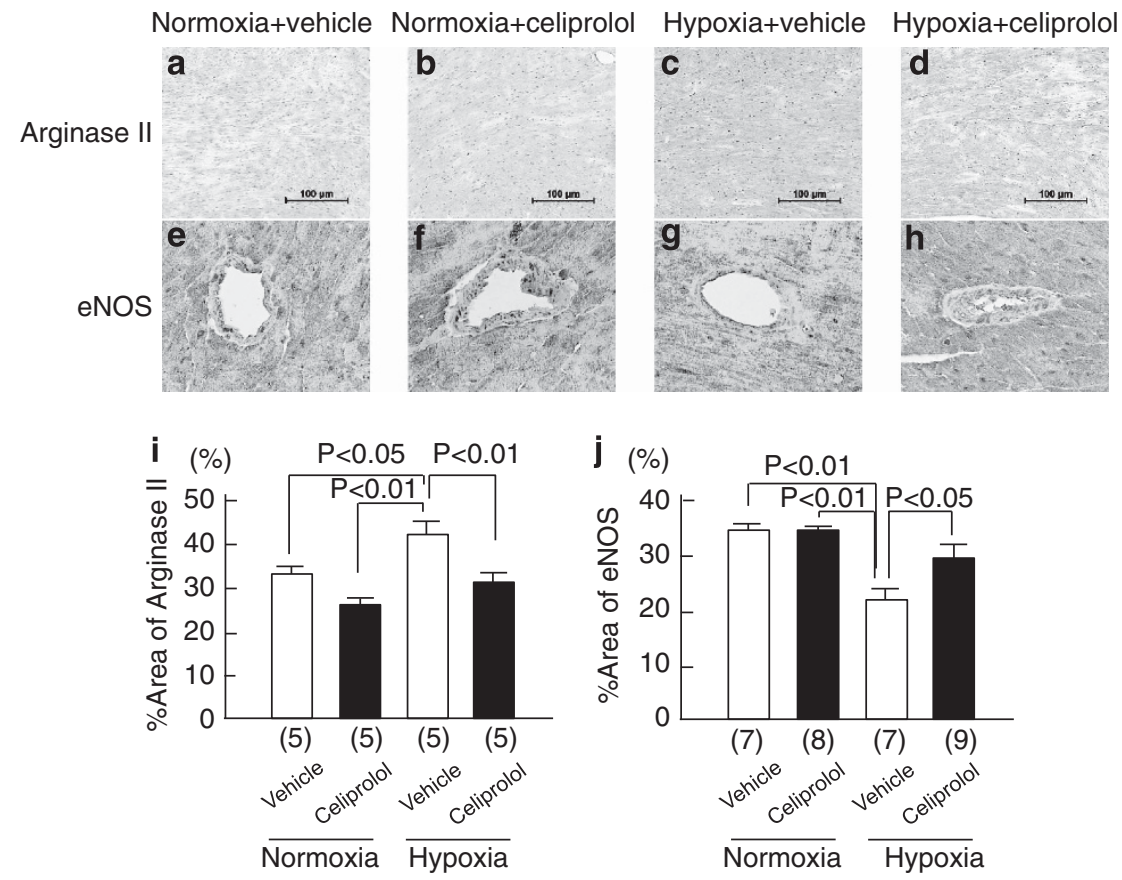

Figure 6 Immunohistochemistry of arginase II (a-d) and eNOS (e-h) expression in the LV myocardium. Arginase II expression (i) was significantly increased and expression of eNOS (j) was significantly decreased by intermittent hypoxia, while this change was suppressed by celiprolol. Values are the mean \pm s.e.m. A full color version of this figure is available at the Hypertension Research journal online.

role in tissue fibrosis and inflammation. In the present study, intermittent hypoxia increased the expression of TNF- $\alpha$ and BNP mRNA in the LV myocardium. Treatment with celiprolol significantly suppressed TNF- $\alpha$ and BNP mRNA expression, and perivascular fibrosis was also significantly reduced. We have previously reported that expression of TGF- $\beta$ mRNA in the LV myocardium was increased in mice treated with hypoxic stress. ${ }^{8}$ It is also reported that celiprolol decreased TGF- $\beta$ in deoxycorticosterone acetate-salt hypertensive rats. ${ }^{29}$ Although we did not investigate TGF- $\beta$ in this study, the anti-inflammatory and antioxidant effects of celiprolol might have a role in lessening the histological changes induced by hypoxic stress. Further investigation will be needed using other $\beta$-blockers (such as propranolol, atenolol and metoprolol) to elucidate the pathophysiology of cardiomyocyte degeneration related to intermittent hypoxia-induced sympathetic activation.

Recently, an angiotensin II receptor blocker (ARB) was reported to inhibit sympathetic nervous system activity in rats. ${ }^{30}$ It has been shown that ARBs exhibit antioxidative and anti-inflammatory effects in human and animals. ${ }^{9,31,32}$ We have reported that combination therapy with celiprolol and candesartan exerts cardioprotective effects 
in heart failure model rats. ${ }^{33}$ We also found that angiotensin II expression was increased by intermittent hypoxia. ${ }^{10}$ Furthermore, olmesartan combined with nifedipine, which has antioxidative effect, was effective to prevent hypoxia-induced cardiac remodeling in diabetic mice. ${ }^{34}$ Therefore, combination therapy with celiprolol and $\mathrm{ARB}$ (or an angiotensin-converting enzyme inhibitor) might be more effective in our experimental model.

In conclusion, the present study showed that intermittent hypoxia increased the cardiomyocyte diameter and perivascular fibrosis, at least partly, in response to an increase in oxidative stress. Celiprolol significantly suppressed BP fluctuations, restored eNOS expression, reduced oxidative stress and decreased superoxide production, consequently attenuating the histological changes caused by hypoxic stress. These findings suggest that treatment with celiprolol might prevent cardiovascular events in borderline hypertensive patients with SAS.

\section{ACKNOWLEDGEMENTS}

This work was supported by a JSPS Kakenhi Grant (Number 23590267) and by Research Funds from Osaka Medical College and the Osaka University of Pharmaceutical Sciences. We are grateful to C Ohta, Y Ogami, Y Mizuoka and $\mathrm{K}$ Shimokawa for their expert technical assistance.

1 Lattimore JD, David S, Wilcox I. Obstructive sleep apnea and cardiovascular disease. J Am Coll Cardiol 2003; 41: 1429-1437.

2 Kato M, Adachi T, Koshino Y, Virend K. Obstructive sleep apnea and cardiovascular disease. Circ J 2009; 73: 1363-1370.

3 Nakashima H, Katayama T, Takagi C, Amenomori K, Ishizaki M, Honda Y. Obstructive sleep apnea inhibits the recovery of left ventricular function in patients with acute myocardial infarction. Eur Heart J 2006; 27: 2317-2322.

4 Alchanatis M, Tourkohoriti G, Kosmas EN, Panoutsopoulos G, Kokouros S, Papadima K, Gaga M, Jordanoglou JB. Evidence for left ventricular dysfunction in patients with obstructive sleep apnea syndrome. Eur Respir J 2002; 20: 1239-1245.

5 Christou K, Markoulis N, Moulas AN, Pastaka C, Gourgoulianis KI. Reactive oxygen metabolites (ROMs) as an index of oxidative stress in obstructive sleep apnea patients. Sleep Breath 2003; 7: 105-110.

6 Ryan S, Taylor CT, McNicholas WT. Selective activation of inflammatory pathways by intermittent hypoxia in obstructive sleep apnea syndrome. Circulation 2005; 112 : 2660-2667.

7 Siwik DA, Tzortzis JD, Pimental DR, Chang DLF, Pagano PJ, Singh K, Sawyer DB, Coucci WS. Inhibition of copper-zinc superoxide dismutase induces cell growth, hypertrophic phenotype, and apoptosis in neonatal rat cardiac myocytes in vitro. Circ Res 1999; 85: 147-153.

8 Hayashi T, Yamashita C, Matsumoto C, Kwak CJ, Fujii K, Hirata T, Miyamura M, Mori T, Ukimura A, Okada Y, Matsumura Y, Kitaura Y. Role of gp91phox-containing NADPH oxidase in left ventricular remodeling induced by intermittent hypoxic stress. Am J Physiol Heart Circ Physiol 2008; 294: 2197-2203.

9 Yamashita C, Hayashi T, Mori T, Tazawa N, Kwak CJ, Nakano D, Sohmiya K, Okada Y, Kitaura Y, Matsumura Y. Angiotensin II receptor blocker reduces oxidative stress and attenuates hypoxia-induced left ventricular remodeling in apolipoprotein E-knockout mice. Hypertens Res 2007; 30: 1219-1230.

10 Matsumoto C, Hayashi T, Kitada K, Yamashita C, Miyamura M, Mori T, Ukimura A, Ohkita M, Jin D, Takai S, Miyazaki M, Okada Y, Kitaura Y, Matsumura Y. Chymase plays an important role in left ventricular remodeling induced by intermittent hypoxia in mice. Hypertension 2009; 54: 164-171.

11 Somers VK, Dyken ME, Clary MP, Abboud FM. Sympathetic neural mechanisms in obstructive sleep apnea. J Clin Invest 1995; 96: 1897-1904.

12 Kakoki M, Hirata Y, Hayakawa $H$, Nishimatsu $H$, Suzuki $Y$, Nagata D, Suzuki E, Kikuchi K, Nagano T, Omata M. Effects of vasodilatory beta-adrenoceptor antagonists on endothelium-derived nitric oxide release in rat kidney. Hypertension 1999; 33: 467-471.

13 Yao EH, Fukuda N, Matsumoto T, Katakawa M, Yamamoto $C$, Han $Y$, Ueno $T$, Kobayashi N, Matsumoto K. Effects of the antioxidative beta-blocker celiprolol on endothelial progenitor cells in hypertensive rats. Am J Hypertens 2008; 21: 12.
14 Mehta JL, Lopez LM, Chen L, Cox OE. Alterations in nitric oxide synthase activity, superoxide anion generation, and platelet aggregation in systemic hypertension, and effects of celiprolol. Am J Cardiol 1994; 7: 901-905.

15 Miller FJ Jr, Gutterman DD, Rios CD, Heistad DD, Davidson BL. Superoxide production in vascular smooth muscle contributes to oxidative stress and impaired relaxation in atherosclerosis. Circ Res 1998; 82: 1298-1305.

16 Nakano D, Hayashi T, Tazawa N, Yamashita C, Inamato S, Okuda N, Mori T, Sohmiya K, Kitarura Y, Okada Y, Matsumura Y. Chronic hypoxia accelerates the progression of atherosclerosis in apolipoprotein E-knockout mice. Hypertens Res 2005; 28: 837-845.

17 Inamoto S, Yoshioka T, Yamashita C, Miyamura M, Mori T, Ukimura A, Matsumoto C, Matsumura Y, Kitaura Y, Hayashi T. Pitavastatin reduces oxidative stress and attenuates intermittent hypoxia-induced left ventricular remodeling in lean mice. Hypertens Res 2010; 33: 579-586.

18 Hayashi T, Yoshioka T, Hasegawa K, Miyamura M, Mori T, Ukimura A, Matsumura Y, Ishizaka N. Inhalation of hydrogen gas attenuates left ventricular remodeling induced by intermittent hypoxia in mice. Am J Physiol Heart Circ Physiol 2011; 301: 1062-1069.

19 Bivalacqua TJ, Hellstrom WJ, Kadowitz PJ, Champion HC. Increased expression of arginase II in human diabetic corpus cavernosum: in diabetic-associated erectile dysfunction. Biochem Biophys Res Commun 2001; 283: 923-927.

20 Morris CR, Morris SM Jr, Hagar W, Van Warmerdam J, Claster S, Kepka-Lenhart D, Machado L, Kuypers FA, Vichinsky EP. Arginine therapy: a new treatment for pulmonary hypertension in sickle cell disease? Am J Respir Crit Care Med 2003; 168: 63-69.

21 Zhang C, Hein TW, Wang W, Miller MW, Fossum TW, McDonald MM, Humphrey JD, Kuo L. Upregulation of vascular arginase in hypertension decreases nitric oxidemediated dilation of coronary arterioles. Hypertension 2004; 44: 935-943.

22 Lopez V, Siques P, Brito J, Vallejos C, Naveas N, Carvallo C, León-Velarde F, Carvajal N. Upregulation of arginase expression and activity in hypertensive rats exposed to chronic intermittent hypobaric hypoxia. High Alt Med Biol 2009; 10: 373-381.

$23 \mathrm{Kim} \mathrm{JH}$, Bugaj LJ, Oh YJ, Bivalacqua TJ, Ryoo S, Soucy KG, Santhanam L, Webb A, Camara A, Sikka G, Nyhan D, Shoukas AA, Ilies M, Christianson DW, Champion HC, Berkowitz DE. Arginase inhibiton restores NOS coupling and reverses endothelial dysfunction and vascular stiffnes in old rats. J App/ Physiol 2009; 107: 1249-1257.

24 Kobayashi N, Mori Y, Nakano S, Tsubokou Y, Shirataki H, Matsuoka H. Celiprolol stimulates endothelial nitric oxide synthase expression and improves myocardial remodeling in deoxycorticosterone acetate-salt hypertensive rats. J Hypertens 2001; 19: 795-801.

25 Mansfield D, Kaye DM, Brunner La Rocca H, Solin P, Esler MD, Nauqhton MT. Raised sympathetic nerve activity in heart failure and central sleep apnea is due to heart failure severity. Circulation 2003; 18: 1396-1400.

26 Bradley TD, Tkacova R, Hall MJ, Ando S, Floras JS. Augmented sympathetic neural response to simulated obstructive apnoea in human heart failure. Clin Sci 2003; 104: 231-238.

27 Hui AS, Striet JB, Gudelsky G, Soukhova GK, Gozal E, Beitner-Johnson D, Gup SZ, Sachleben LR Jr, Haycock JW, Gozal D, Czyzyk-krzeska MF. Regulation of catecholamines by sustained and intermittent hypoxia in neuroendocrine cells and sympathetic neurons. Hypertension 2003; 42: 1130-1136.

$28 \mathrm{Li} \mathrm{S}$, Qian XH, Zhou W, Zhang Y, Feng J, Wan NS, Zhang Z, Guo R, Chen BY. Time-dependent inflammatory factor production and NFkB activation in a rodent model of intermittent hypoxia. Swiss Med Wkly 2011; 141: 13309-13320.

29 Kobayashi N, Mita S, Yoshida K, Honda T, Kobayashi T, Hara K, Nakano S, Tsubokou Y, Matsuoka H. Celiprolol activates eNOS through the PI3K-Akt pathway and inhibits VCAM-1 Via NF-kB induced by oxidative stress. Hypertension 2003; 42: 1004-1013.

30 Konno S, Hirooka Y, Kishi T, Sunagawa K. Sympathoinhibitory effects of telmisartan through the reduction of oxidative stress in the rostral ventrolateral medulla of obesity-induced hypertensive rats. J Hypertens 2012; 30: 1992-1999.

31 Taguchi I, Toyoda S, Takano K, Arikawa T, Kikuchi M, Ogawa M, Abe S, Node K, Inoue T. Irbesartan, an angiotension receptor blocker, exhibits metabolic, antiinflammatory and antioxidative effects in patients with high-risk hypertension. Hypertens Res 2013; 36: 608-613.

32 Inamoto S, Hayashi T, Tazawa N, Mori T, Yamashita C, Nakano D, Matsumura Y, Okuda N, Sohmiya K, Sakai A, Furuya E, Kitaura Y. Angiotensin II receptor blocker exerts cardioprotection in diabetic rats exposed to hypoxia. Circ J 2006; 70: 787-792.

33 Mori T, Hayashi T, Sohmiya K, Okuda N, Shimomura H, Ohkita M, Matsumura Y, Yoshiyama M, Yoshikawa J, Kitaura Y. Mechanisms of combined treatment with celiprolol and cabdesartan for ventricular remodeling in experimental heart failure. Circ J 2005; 69: 596-602.

34 Yamashita C, Hayashi T, Mori T, Matsumoto C, Kitada K, Miyamura M, Sohmiya K, Ukimura A, Okada Y, Yoshioka T, Kitaura Y, Matsumura Y. Efficacy of olmesartan and nifedipine on recurrent hypoxia-induced left ventricular remodeling in diabetic mice. Life Sci 2010; 86: 322-330. 